\title{
Señalización y seguridad vial en buses de tránsito rápido: el transmilenio en Bogotá
}

\section{Road signage and road safety in bus rapid transit corridors: transmilenio in Bogotá}

\section{Yefer Asprilla Lara}

Universidad Distrital Francisco José de Caldas, Colombia, estudiante Doctorado en Movilidad Urbana, Transporte y Territorio, Centro Universitario de Tonalá-Universidad de Guadalajara

yeasla@hotmail.com
Fernando García De Quevedo

Instituto Tecnológico de Monterrey Campus Guadalajara, México

fdoquevedo@itesm.mx

Mario Gpe. González Pérez

Universidad de Guadalajara, México

inge_united@hotmail.com

Fecha de recepción: 20 de noviembre de 2016 / Fecha de aprobación: 20 de julio de 2017

\section{RESUMEN}

El incremento de la accidentalidad vial en la mayoría de los países del mundo ha encendido las alarmas de la práctica de la movilidad urbana e interurbana contemporáneas. Los percances, ocurren tanto en sistemas de transporte público, como en vehículos privados o sistemas masivos de transporte. En el presente escrito, se estudia la influencia de la señalización en la seguridad vial de los usuarios del sistema Transmilenio en el eje troncal de la avenida Caracas en Bogotá, Colombia, a través del análisis descriptivo y la inspección in situ. Los resultados arrojan que es impostergable para los gobiernos nacionales y municipales implementar políticas de mitigación y medidas orientadas a subsanar los efectos socioeconómicos y psicológicos generados por la práctica de la movilidad urbana motorizada.

PALABRAS CLAVE: Señalización, seguridad vial, accidentalidad, buses de tránsito rápido.

\begin{abstract}
The increase in traffic accidents in most countries of the world has triggered the alarms in urban and interurban mobility safety. Accidents occur both in public transport systems, such as private vehicles or mass transit systems. In this paper, the influence of road signage on users' road safety of the Transmilenio system in the main arterial of the Caracas Avenue in Bogotá, Colombia is studied through descriptive analysis and on-site inspection. The results show that it is urgent that national and municipal governments implement mitigation policies and measures aiming at decreasing socioeconomic and psychological effects generated by motorized traffic.
\end{abstract}

KEYWORDS: Road signage, road safety, traffic accidents, Bus Rapid Transit. 


\section{INTRODUCCIÓN}

Las diferentes investigaciones realizadas en seguridad vial convergen en que el factor humano, con todas sus implicaciones sociales, el vehículo y la infraestructura son los ejes en donde podría tener origen la accidentalidad vial. Efectivamente, los accidentes de tránsito no son solo un problema de señalización o educación vial, sino que se han convertido en un tema de salud pública, que afecta a todos los países del mundo, pero especialmente aquellos con ingresos medios y bajos (Chan, 2011). Inclusive, la Organización Mundial de la Salud (2015) argumenta que cerca de 1,25 millones de personas perecen anualmente y alrededor de 50 millones más, quedan lesionadas en las vías urbanas e interurbanas de los núcleos poblacionales. Por lo cual, la seguridad vial ha sido estudiada y analizada desde hace años por diferentes organizaciones e instituciones a nivel global como la Organización Mundial de la salud (OMS), la Organización Panamericana de la Salud y el Banco Mundial; y en el caso de los sistemas de autobuses de tránsito rápido, organismos como el Instituto de Políticas para el Transporte y Desarrollo, el Centro de Transporte Sostenible del Instituto de Recursos Mundiales, la Asociación Latinoamericana de Sistemas Integrados y BRT, y el Fondo de Prevención Vial. Incluso se estima que de no tomar en cuenta las medidas pertinentes en materia de seguridad vial, para 2030 los decesos por accidentes de tránsito pasarían a ser la quinta causa de muerte a nivel global, después de las enfermedades pulmonares obstructivas crónicas e infecciones por vías respiratorias (OMS, 2015).

Ciertamente, el tema de la seguridad vial en los sistemas masivos de transporte público es un caso particular; sobre todo en aquellos sistemas de autobuses de tránsito rápido (BRT), los cuales existen en un poco más de 200 ciudades (Global BRT Data, 2016), y se han convertido en una de las soluciones mayormente implementadas en países latinoamericanos $y$ asiáticos, por sus beneficios socioeconómicos y ambientales en la práctica de la movilidad. El BRT (Bus Rapid Transit) es un sistema de transporte público que se caracteriza por no mezclarse con el tráfico mixto. Es decir, el tránsito ocurre por un carril exclusivo. En 1974, la ciudad de Curitiba en Brasil, fue la primera en implementar este sistema, luego en diciembre del 2000 lo adopto Bogotá, con el denominado sistema Transmilenio (Lindau et al. 2010). Este sistema se ha caracterizado por una serie de dificultades como: baja frecuencia de rutas, bloqueos, ventas ambulantes, accidentalidad, "colados" (acceso al sistema sin pago del pasaje), entre otras, que han afectado notoriamente la calidad del servicio. Además, según datos de la Dirección de Seguridad Vial de la Secretaría Distrital de Movilidad (2016), se tiene evidencia que en los últimos cinco años el problema de la accidentalidad ha sido continuo (Véase Figura 1).
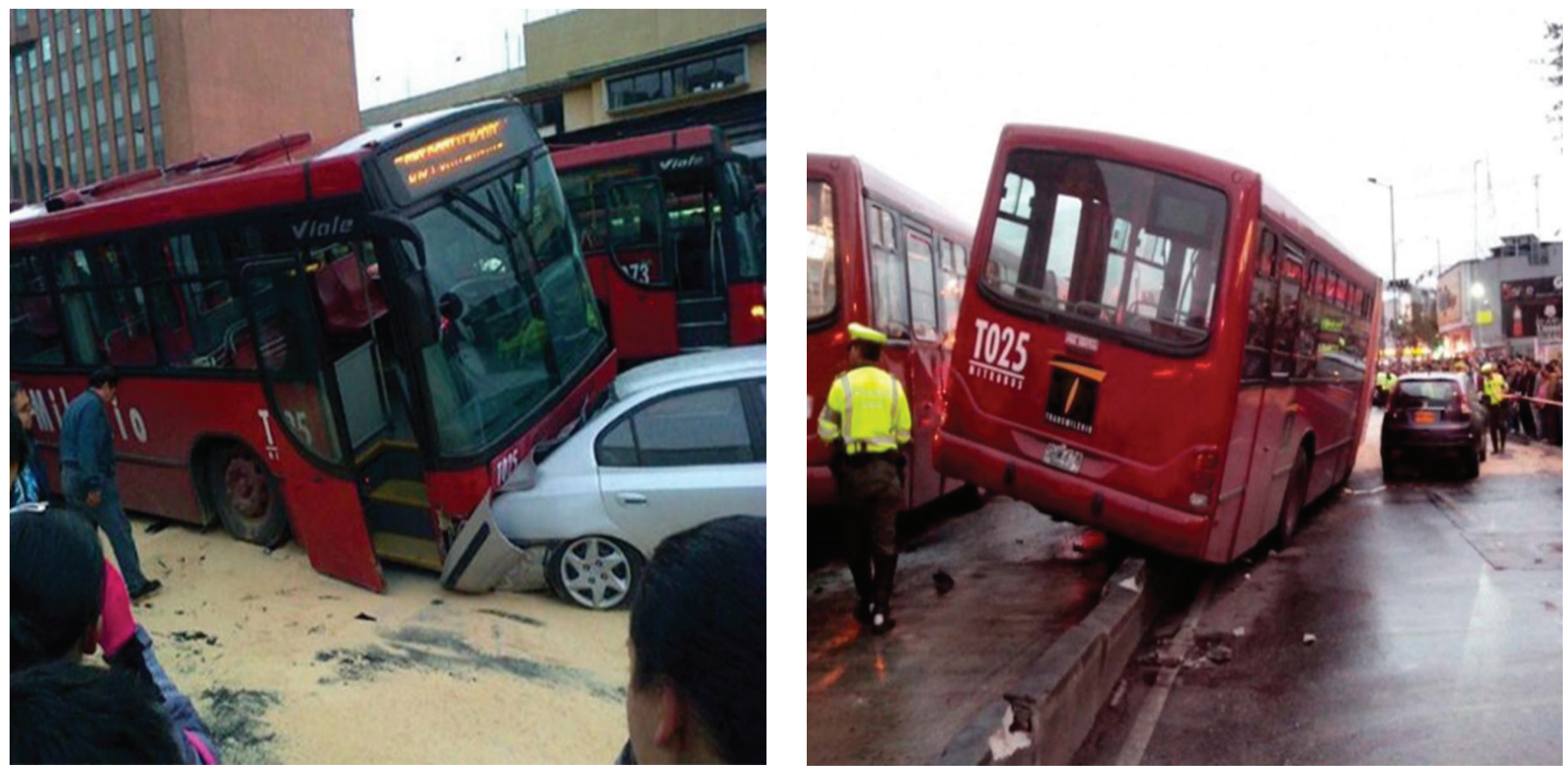

Figuras 1. Accidente de BRT Transmilenio en la Av. Caracas Fuente:@kmiilowm en El Universal, 2013 
Efectivamente, el año 2014 registró un total en el sistema Transmilenio de 470 accidentes, con 24 decesos y 540 lesionados, y en el 2015 se redujo $22,1 \%$ el número de accidentes, con $26,4 \%$ menos lesionados; pero no así, el número de fallecimientos, que pasaron de 24 a 27 personas (Secretaria Distrital de Movilidad de Bogotá, 2016). Esta situación podría estar relacionada con el incremento de la demanda de pasajeros y, en consecuencia, por mayores operaciones y crecimiento de la flota de autobuses. A su vez, se adhiere la entrada en operación de la fase III y la extensión del servicio al municipio de Soacha; sobre todo porque después de 12 años de operación de la fase I: troncal avenida Caracas, calle 80, autopista y la Fase II: troncales Norte-Quito sur, Suba y Américas. Sin embargo, en 2012 entra la Fase III del sistema Transmilenio, la cual comprende las troncales de la carretera 10 y calle 26 (Transmilenio, 2014).
En este contexto, un comparativo de los distintos tipos de accidentes que ocurrieron en el sistema Transmilenio entre los años 2013 y 2014, con Informe Policial de Accidente de Tránsito (IPAT), se logra evidenciar que los atropellos a peatones se incrementaron en un 53\%, en ciclistas el incremento fue del $48 \%$ y en los choques con motociclista el incremento fue del 50\%; lo anterior evidencia que la frecuencia de los accidentes se presenta con los usuarios más vulnerables de la movilidad, lo que permite inferir que en la ocurrencia de estos eventos, exista alguna falla de los elementos que conforman la infraestructura del sistema, como señalización y otros. Sin embargo, las hipótesis atribuidas a los accidentes asociadas con los buses del sistema Transmilenio muestran que en el 47,5\% de los casos se desconocen sus causas, y al resto se le relaciona con la operación en la prestación del servicio, el tránsito, la señalización, entre otras (Véase Figura 2).

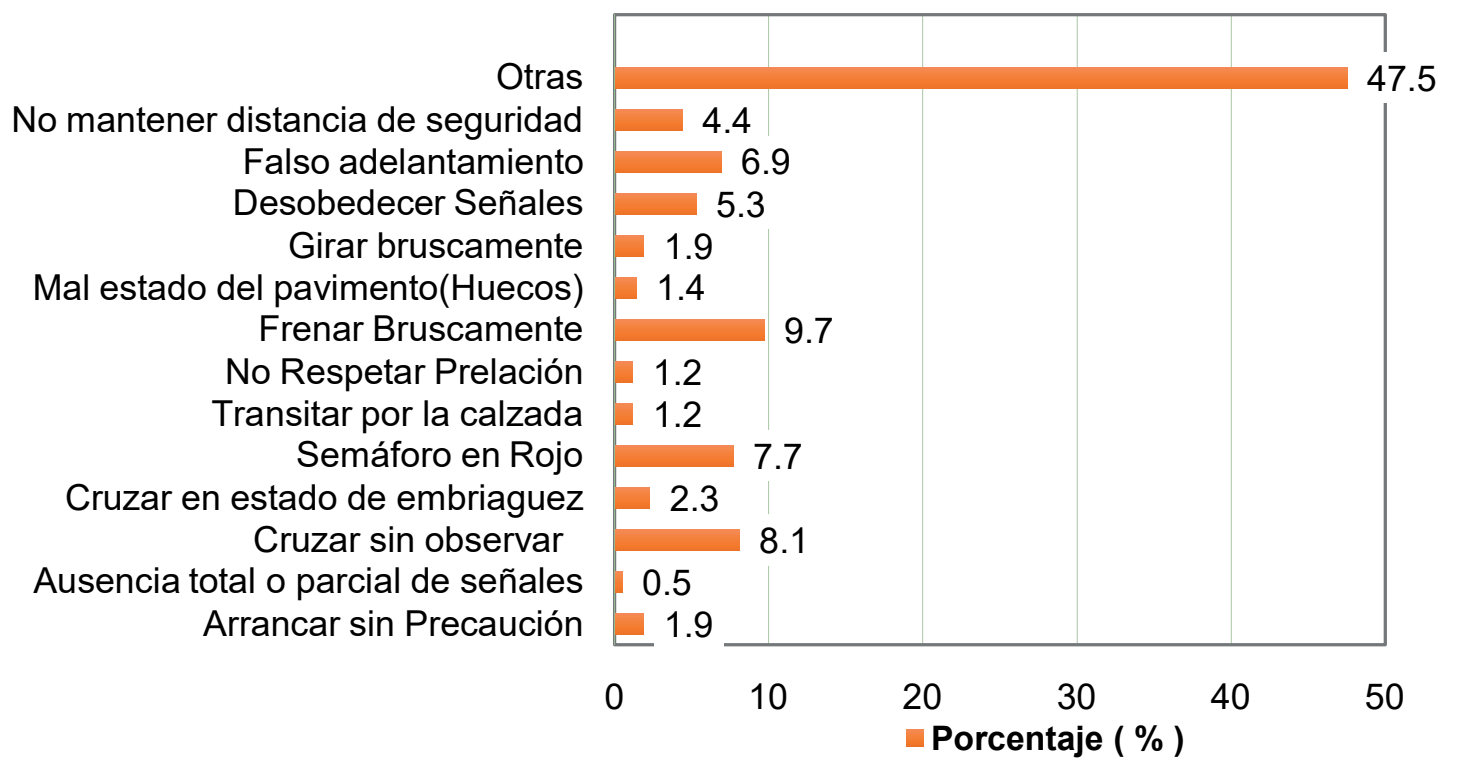

Figura 2. Principales hipótesis de los accidentes del sistema Transmilenio Fuente: Secretaria Distrital de Movilidad de Bogotá, 2016

Las principales causas o hipótesis de los accidentes que se muestran en la figura anterior como: frenar bruscamente, cruzar semáforo en rojo, cruzar sin observar, desobedecer las señales de tránsito, adelantar cerrando o entre cruzamientos, no mantener distancia de seguridad entre otras; se evidencian en los tipos de accidentes más frecuentes que se presentaron en el sistema durante el periodo 2013-2014, entre las que se resaltan las caídas dentro del vehículo, seguido por atropellos a peatones y ciclistas, luego choques con terceros, choques entre buses articulados y motociclistas, lo cual da cuenta que los accidentes presentados en el sistema interactúan los diferentes modos de la movilidad cotidiana, siendo los más afectados los usuarios y actores vulnerables como peatones y ciclistas (Véase Figura 3). 


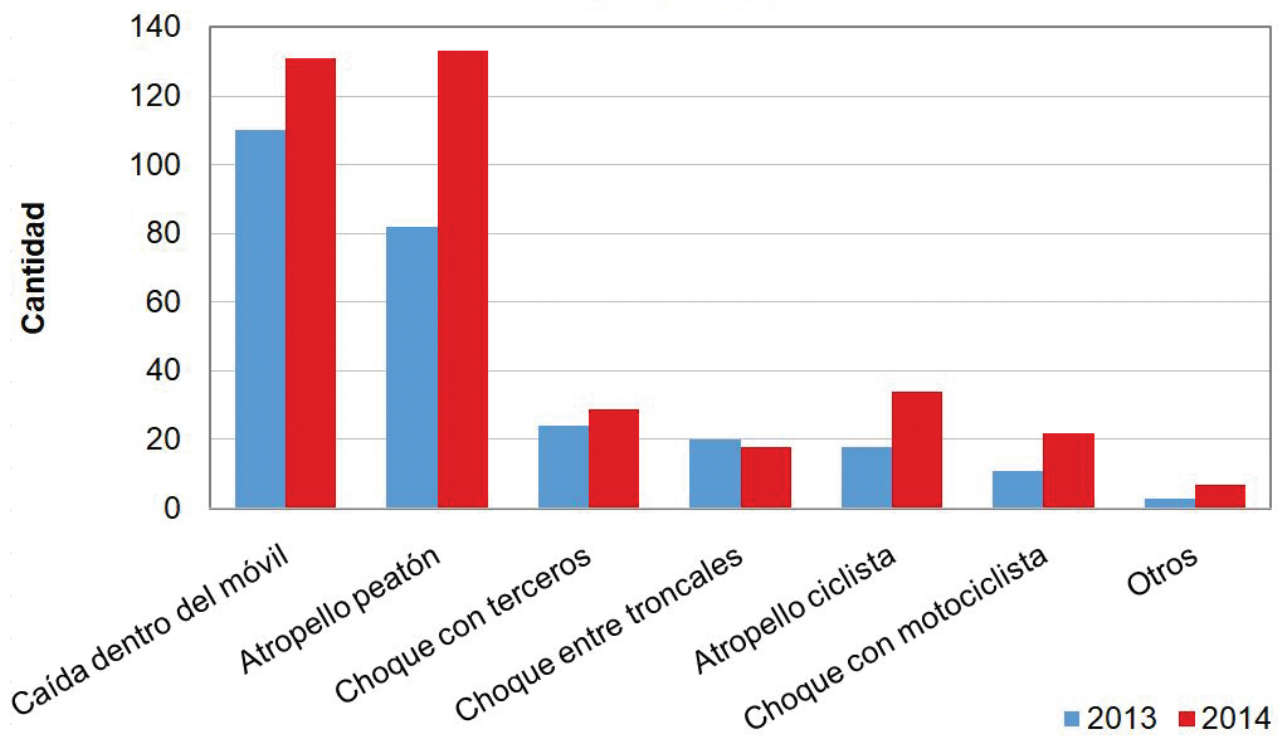

Figura 3. Tipo de eventos (accidentes) frecuentes en el sistema Transmilenio. Fuente: Informe de Gestión Sistema Transmilenio, 2014

El presente estudio está circunscrito dentro del "factor infraestructura"; pues suponemos que en la medida que esta incorpore los mecanismos necesarios para la buena práctica de la movilidad, se estaría en condiciones de contribuir con la reducción de los indices de accidentalidad. Por lo que se cuestiona: ¿De qué forma incide la señalización en los percances de tránsito?, ¿qué características presenta la señalización de la infraestructura utilizada por el sistema Transmilenio?, ¿qué expectativas se generan en materia de seguridad vial en el sistema Transmilenio? El objetivo central consiste en analizar la influencia de la señalización en la seguridad vial del sistema BRT Transmilenio, uno de los más reconocidos a nivel mundial (Duarte y Rojas, 2012; Lindau et al. 2010), considerando exclusivamente la señalización vertical y horizontal del eje troncal de la Avenida Caracas. Para ello, se empleó el método analítico descriptivo aunado con la inspección visual in situ y apoyado con imágenes de Google Earth.

\section{MARCO TEÓRICO Y METODOLÓGICO}

\section{Los BRT: un breve bosquejo}

Los primeros sistemas BRT fueron propuestos por Jaime Lerner en la década de los setentas en Curitiba, Brasil. Por esos años, se daba un debate en torno a la construcción de un sistema férreo de tren ligero, o LRT (por sus siglas en inglés), como opción para resolver los problemas de movilidad en la ciudad. Sin embargo, por las dificultades económicas y falta de apoyo financiero, el proyecto no se pudo materializar; lo cual condujo a que la administración municipal buscara alternativas que cumplieran las mismas funciones de los sistemas férreos en términos de eficiencia y rapidez, pero a menores costos por kilómetro y tiempo de construcción (Lindau et al. 2010).

A partir de este evento nacen los sistemas BRT, con particularidades que permitieran cumplir con las expectativas del momento, como la preferencia de circulación por carriles exclusivos y la integración con otros sistemas de transporte. Esto inspiró, más luego que tarde para que otras ciudades de Latinoamérica se apropiaran de este modelo de transporte (Duarte y Rojas, 2012). En el caso de la ciudad de Bogotá, desde finales de la década de los noventas, los problemas de movilidad urbana y la falta de calidad en la prestación del servicio de transporte público como los largos tiempos de los viajes, las bajas velocidades por la congestión vehicular, desorganización de rutas con sobre ofertas en algunos sitios y baja cobertura en otros, accidentalidad, contaminación ambiental, caos en la prestación del servicio y una frágil normatividad, condujeron a la realización de un estudio sobre el sistema de transporte y la movilidad de la ciudad (Asprilla y Rey, 2012).

Con la carta de navegación trazada, se analizaron y evaluaron diferentes alternativas de sistemas masivos o semi-masivos de transporte público de pasajeros de otras ciudades latinoamericanas y países desarrollados. Como consecuencia, la administración distrital de ese entonces, decidió adaptar e implementar a finales del año 2000, con algunos ajustes, el modelo exitoso de transporte de Curitiba, el sistema denominado Transmilenio. 
En la actualidad, los sistemas BRT están presentes en cerca de 203 ciudades a nivel global y mueven más de treinta y tres millones de pasajeros por día. Goblal brt data por ejemplo, es una plataforma pública para compartir datos de sistemas prioritarios de autobús, administrado por WRI-Brasil, la cual recoge, procesa y almacena los datos e información fiable y actual de todos los sistemas BRT que existen en el mundo, brindando información actualizada de las 203 ciudades que hoy cuentan con este sistema masivo de transporte público (Global BRT Data, 2016). En Colombia, los resultados positivos mostrados desde un inicio por el sistema Transmilenio, posibilitaron la réplica (con algunas modificaciones), en otras seis ciudades importantes del país (Pereira, Bucaramanga, Cali, Barranquilla, Medellín y Cartagena). No obstante, en materia de accidentalidad, se han estado presentando percances en los ejes viales adecuados para estos sistemas de transporte, lo cual llevó al Fondo de Prevención Vial (Actualmente la Agencia Nacional de Seguridad Vial), a publicar en 2014 la "Guía de lineamientos en seguridad vial y peatonal para diseño y operación de sistemas de transporte masivo en buses", y ha servido de orientación para los sistemas BRT actualmente en operación, debido a que integra diferentes factores relacionados con la seguridad vial, tales como: humano, vía-entorno y vehículo, con una visión holística de interrelación e interdependencia para una mejor seguridad.

\section{Señalización y seguridad vial}

Dentro del "factor vía-entorno", la señalización ha tenido una relevancia global desde mediados del siglo XX, pues es vista como un elemento fundamental que contribuye con la seguridad vial. En este sentido, diversas organizaciones internacionales y nacionales se han dado a la tarea de realizar estudios sobre la generalización y unificación de las señales y simbologías en materia de tránsito (Organización de Naciones Unidas, 1949,1955; Organización de Estados Americanos, 1991; Ministerio de Transporte de Colombia, 2004; 2014 y Secretaria de Comunicaciones y Transporte de México, 2014).

En Colombia, recientemente se expidió el Manual de señalización vial: Dispositivos uniformes para la regulación del tránsito en calles, carreteras y ciclorutas de Colombia, y de acuerdo con el “inciso e”, “Artículo 2" de la Ley 105 de 1993 y “artículo 2" de la Ley 336 de 1996, se estableció la clasificación y definición de las señales de tránsito, las cuales tienen como función indicar al usuario de las vías sobre las precauciones que debe tener en cuenta, las restricciones y liberaciones que se rigen en el tramo de circulación. Asimismo, también abarca la información necesaria para carriles exclusivos de sistemas BRT, incluyendo los últimos avances tecnológicos que se han desarrollado alrededor del mundo en materia de señalización vial (Ministerio de Transporte, 2014). Diferentes investigaciones han demostrado que la señalización influye en la seguridad y reducción de los accidentes viales; las señales de advertencia, por ejemplo, pueden reducir los accidentes hasta un 20\% (Tignor, 1999), o bien la utilización de semáforos en intersecciones y cruces peatonales podría reducir el número de accidentes alrededor de un 15\% en intersecciones en forma de $\mathrm{T}$ y cerca de un 30\% en los cruces (Rune et al. 2013).

En el caso de la señalización en los sistemas BRT, esta constituye el pilar fundamental para la comunicación entre la infraestructura, conductores y usuarios. Una deficiencia de esta, puede resultar en confusiones para conductores y peatones y afectaciones a las velocidades de operación, lo que conlleva una mayor vulnerabilidad al sistema (Fondo de Prevención Vial, 2014). En este sentido, las señales deben ser necesarias, visibles y llamar la atención. De igual forma, deben ser legibles y de fácil entendimiento, para dar tiempo suficiente al usuario de la movilidad y este pueda responder adecuadamente. Finalmente, deben infundir respeto y ser creíbles (Ministerio de Transporte, 2004).

En algunos corredores viales de los sistemas BRT, los cuales tienen como característica principal estar segregados de otros actores de la movilidad, la señalización no cumple con los requisitos de visibilidad, legibilidad, comprensibilidad, credibilidad e infundir respeto. Esto conlleva, a que algunos usuarios de las vías las incumplan y pongan en riesgo su vida al circular por los carriles exclusivos de los sistemas BRT. Por otro lado, la señalización horizontal o marcas en el pavimento (cruces peatonales, cebras, marcas solo bus, separadores, flechas, etc.), en algunos casos son relegadas por las autoridades municipales gestoras los sistemas BRT.

\section{Descripción del corredor troncal}

El corredor de la Avenida Caracas es la principal línea troncal del sistema Transmilenio, y está dividido en dos segmentos: troncal Caracas y trocal Caracas sur. Este último inicia en la parte sur de la localidad de Usme y termina en la parte norte de la localidad de Chapinero (Véase Figura 4). 


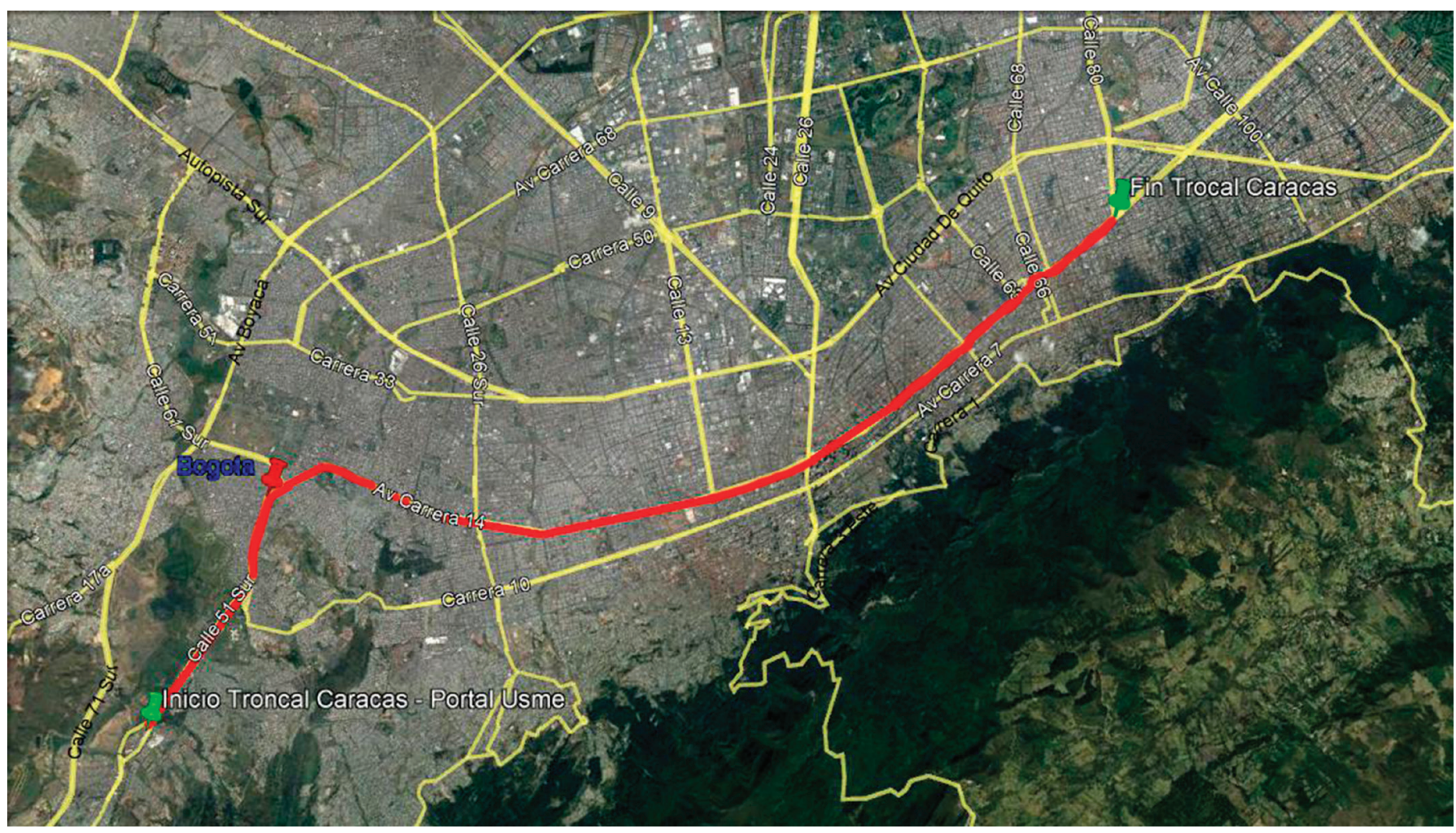

Figura 4. Corredor troncal Avenida Caracas.

Fuente: Google Earth, 2016

Las características infraestructurales del corredor de estudio (Véase Cuadro 1), hace parte de la malla vial arterial de la ciudad y es uno de los que soporta la movilidad y accesibilidad urbana en largas distancia. Esta troncal tiene una longitud total de 22 kilómetros, un ancho de 60 metros incluyendo la acera para los peatones, dos carriles vehiculares en cada sentido para el tráfico mixto, los cruceros son regulados mediante sincronización semafórica y en esta línea el sistema cuenta con 2 estaciones intermedias y 24 sencillas ubicadas entre los 500 y 700 metros de distancia.

Cuadro 1. Características de la infraestructura del corredor troncal avenida Caracas sistema Transmilenio desde el portal Usme a la estación de calle 76

\begin{tabular}{|c|c|c|c|}
\hline Características & Cantidad & $\begin{array}{l}\text { Ancho } \\
\text { (metros) }\end{array}$ & $\begin{array}{l}\text { Longitud total } \\
\text { (Kilómetros) }\end{array}$ \\
\hline Longitud total del corredor & 1 & 40 & 22 \\
\hline Calzadas segregadas por sentido & 2 & 7 & 19.2 \\
\hline Carriles segregados y exclusivos por sentido & 2 & 3.5 & 19.2 \\
\hline Carriles tráfico mixto por sentido & 2 & 3.9 & 22 \\
\hline $\begin{array}{l}\text { Intersecciones vehiculares y reguladas semafóricamente (distancia prome- } \\
\text { dio entre ellas) }\end{array}$ & 56 & 7 a 40 & 0.392 \\
\hline Total Intersecciones reguladas por semáforos & 62 & 4 a 40 & 0.354 \\
\hline
\end{tabular}


Según datos de Transmilenio (2014), en el día se movilizan cerca de 521.000 pasajeros y en horas pico hasta 48.000 pasajeros/ hora/sentido; el sistema opera en corredores segregados del tráfico mixto, por lo tanto, en el tramo de estudio la circulación de los autobuses es en calzadas exclusivas, con una intensidad del tráfico en hora pico de 320 buses/hora aproximadamente, lo que representa una frecuencia promedio de 5.3 buses/ minutos, lo que indica una separación entre buses de tan solo 11.3 segundos.
Esto llevaría a que las características de la señalización vertical como horizontal requieran tener estándares mucho más altos a los que existen actualmente. Por otro lado, el crecimiento del tráfico de los autobuses en el corredor de estudio ha sido progresivo, a la par con el crecimiento de la demanda de pasajeros del sistema, la cual en los últimos años ha crecido en promedio 7\% (Véase Cuadro 2).

Cuadro 2. Crecimiento promedio del tráfico de buses/hora punta, troncal de estudio

\begin{tabular}{|c|c|c|c|c|c|c|}
\hline Años & 2010 & 2011 & 2012 & 2013 & 2014 & 2015 \\
\hline Tráfico promedio (buses/hora punta ) & 210 & 225 & 228 & 272 & 295 & 320 \\
\hline
\end{tabular}

Este crecimiento del tráfico de buses y de la demanda ha generado que en esta troncal se concentre el mayor número de accidentes viales de todo el sistema, lo cual se ubica en un rango entre 30 a $35 \%$. En el periodo comprendido entre 2010 y 2015, los accidentes del sistema se incrementaron por múltiples causas, una de ellas relacionada con señalización.

Al hacer un análisis de los accidentes ocurridos durante el periodo 2014-2015 en este corredor troncal, teniendo en cuenta la hora del día, encontramos que la mayor concentración se presenta en los periodos de horas picos de la mañana, medio día y noche. Lo anterior permite inferir que por el aumento de las operaciones (buses/hora) debido a la demanda de pasajeros, las distancias de separación entre buses y visibilidad de las señales disminuyen, lo que genera un mayor riesgo de accidentes en estas franjas horarias (Véase Figura 5).

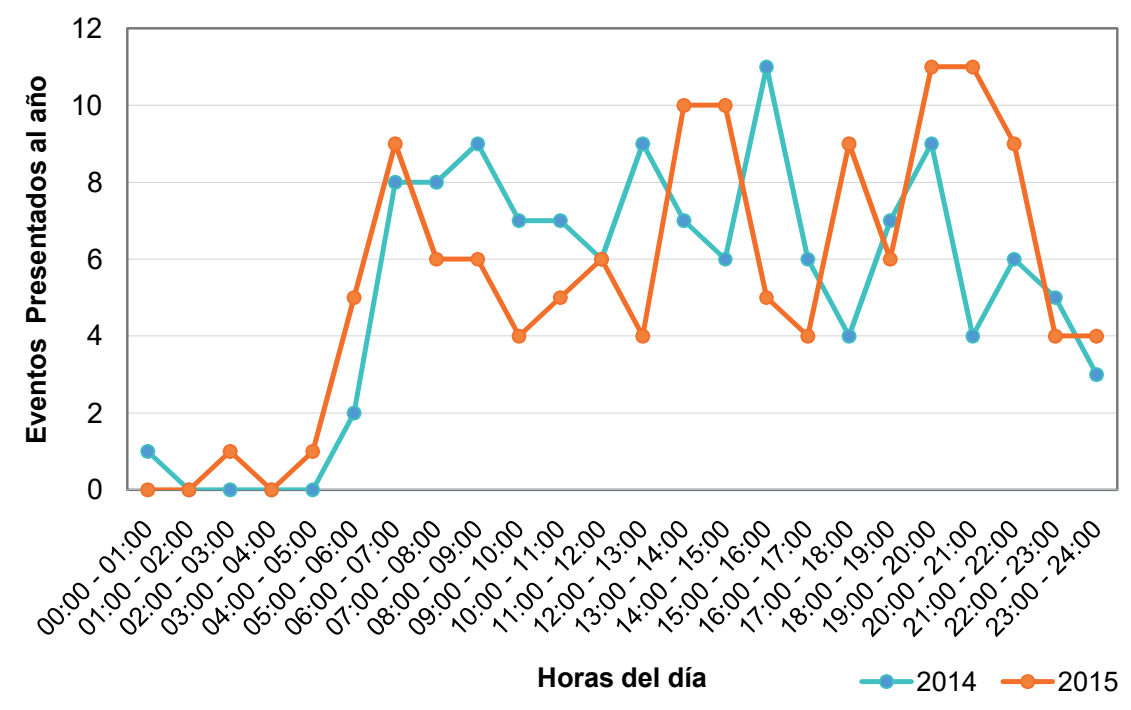

Figura 5. Accidentes por horas del día, corredor troncal avenida Caracas. Fuente: Secretaria Distrital de Movilidad de Bogotá, 2016

\section{Procedimiento metodológico}

Para realizar el análisis de la influencia de la señalización en la seguridad de los sistemas BRT, se realizó una inspección visual, haciendo énfasis en el sistema Transmilenio de la ciudad de Bogotá, Colombia. Para ello, se tomó como referencia el corredor troncal más importante y el que más presenta accidentes: la avenida Caracas. La inspección requirió la utilización de imágenes de Google Earth, y se efectuó un recorrido por todo el eje de estudio, para evaluar las cuatro características que se asume debe cumplir la señalización vial (visibilidad, legibilidad, comprensión, credibilidad). La escala de valoración cualitativa comprendió calificaciones de 1 a 5 puntos y detalladas en orden descendente (Véase Cuadro 3). 
Cuadro 3. Escala de valoración por inspección visual del estado de la señalización

\begin{tabular}{|c|c|}
\hline Valoración del estado de la seîalización & Calificación (puntos) \\
\hline Muy Buena & 5 \\
\hline Buena & 4 \\
\hline Regular & 3 \\
\hline Deficiente & 2 \\
\hline Mala & 1 \\
\hline
\end{tabular}

La calificación numérica de la señalización, que se muestra en la tabla anterior, tomó como referencia las características utilizadas por International Road Assessment Program - IRAP (Programa Internacional de Evaluación de Carreteras), la cual utiliza criterios cualitativos para evaluar los elementos que influyen en la seguridad vial, como lo es la señalización; este proceso es soportado bajo la revisión o inspección visual realizada in situ. La valoración utilizada por IRAP para evaluar la señalización se basa en tres categorías de calificación: buena, mala o regular; en nuestra investigación adicionamos dos categorías más que consideramos pertinentes, debido a que se hace una evaluación más ajustada a la realidad urbana del corredor de estudio. Una señalización muy buena cumple con los cuatro criterios de visibilidad, legibilidad, comprensión, credibilidad en la troncal de la avenida Caracas e influye de manera positiva en la seguridad vial para todos los actores de la movilidad que interactúan con el sistema de Transmilenio. Una señalización mala no cumple con ningunos de los criterios que debe tener la señalización vial, lo cual induce a cometer errores y generación de eventos viales entre los diferentes modos de transporte, especialmente los buses articulados y biarticulados del sistema masivo de pasajeros tipo BRT.

\section{RESULTADOS}

Para Jimeno (2013), las señales deben cumplir cuatro aspectos básicos como son: visibilidad, legibilidad, comprensibilidad y credibilidad. Estas premisas han sido contempladas en el "Manual de señalización vial: Dispositivos uniformes para la regulación del tránsito en calles, carreteras y ciclorutas de Colombia 2015" y el cual nos ha servido de fundamento para el análisis de la señalización en el sistema BRT Transmilenio. Sobre la premisa "visibilidad", se tienen dos categorías: una visibilidad fisiológica, que tiene que ver con la máxima distancia a la cual se puede leer el mensaje de una señal, y se estima en 800 veces la altura de la letra o símbolo. Pero, por otro lado, está la visibilidad geométrica la cual se define como la máxima distancia medida sobre la vía en la que la visual del conductor hacia la señal esté libre de obstáculos (Jimeno, 2013). Sin embargo, esto no ocurre en la visibilidad de las señales verticales instaladas en la troncal de la avenida Caracas del sistema Transmilenio. La legibilidad entonces, busca que la señalización sea legible a cierta distancia, según el Manual de Señalización de Colombia, la distancia de legibilidad mínimo es 400 metros en vías urbanas. Sobre todo, porque distancia y luminancia de una señal son factores fundamentales desde la perspectiva de la lectura, estos tienen directa relación con el manejo de información al volante y los requisitos tipográficos no deben inducir confusión al conductor. Es decir, la legibilidad apunta al reconocimiento de las letras y símbolos de una señal (Gallardo, 2014). No obstante, se evidencian poca o nula legibilidad de las marcas en el pavimento de cebras y líneas separadoras de carriles en el corredor de la troncal de la Avenida Caracas.

Existe coincidencia en torno a que una señalización no debe exigir esfuerzos de comprensión y atención. Es decir, el individuo o usuario de la vía debe asimilar de manera instantánea la información del estímulo visual de un medio exterior; inclusive dentro de un entorno de contaminación visual (Márquez, 2011). En este entendido, la comprensibilidad, va a estar en función del diseño y forma de la señal horizontal o vertical, de tal manera que proporcione el tiempo suficiente para comprender lo que quiere decir y responder adecuadamente. Por su parte, la credibilidad se soporta en el respeto hacia todos los usuarios viales. Esto implica entregar mensajes verdaderos y confiables, sin caer en contradicciones o ambigüedades que lleven a desinformar y confundir al actor móvil. De ahí que, para acatar y obedecer los señalamientos es importante que el tamaño, forma y mensaje concuerden con la situación que se señaliza. Sin embargo, esto no siempre ocurre así, la evaluación de cada uno de los criterios analizados en el estudio de la señalización vertical y horizontal obtuvo que la mejor calificación fue para la comprensibilidad con 3,7 puntos, seguida por la visibilidad y legibilidad con 3,5 puntos. Esto demuestra que la señalización del corredor es comprensible pero tiene poca credibilidad (Véase Cuadro 4). 
Cuadro 4. Evaluación de la señalización, troncal Avenida Caracas, Transmilenio.

\begin{tabular}{|c|c|c|c|c|c|}
\hline \multirow{2}{*}{ Señalización } & \multicolumn{4}{|c|}{ Caracteristicas de la señalización } & \multirow{2}{*}{ Total } \\
\cline { 2 - 6 } & Visibilidad & Legibilidad & Compresibilidad & 3,5 & 3,7 \\
\hline Vertical & 4,0 & 3,5 & 3,8 & 3,2 & 3,2 \\
\hline Horizontal & 3,0 & 3,5 & 3,5 & 3,4 & 3,48 \\
\hline Total & 3,5 & 3,5 & 3,7 & 3 \\
\hline
\end{tabular}

Se puede observar además que la señalización vertical resulta mejor calificada frente a la señalización horizontal, en todos los criterios considerados. En este sentido, el estado en que se encuentra la señalización del corredor troncal de la avenida Caracas obtiene una calificación de 3,48 puntos de 5 puntos posibles, y es equivalente a una valoración regular, cercana a aceptable (Véase Figura 6).
La calificación obtenida evidencia que la señalización del corredor de estudio no es buena y analizando las causas de los accidentes, combinado con los tipos de eventos que se han presentado en los últimos años; se infiere que la señalización ha influido de alguna manera en la ocurrencia de los lamentables hechos viales, debido a que tiene regular visibilidad, legibilidad, comprensibilidad y credibilidad.

\section{Evaluación Señalización BRT Transmilenio}

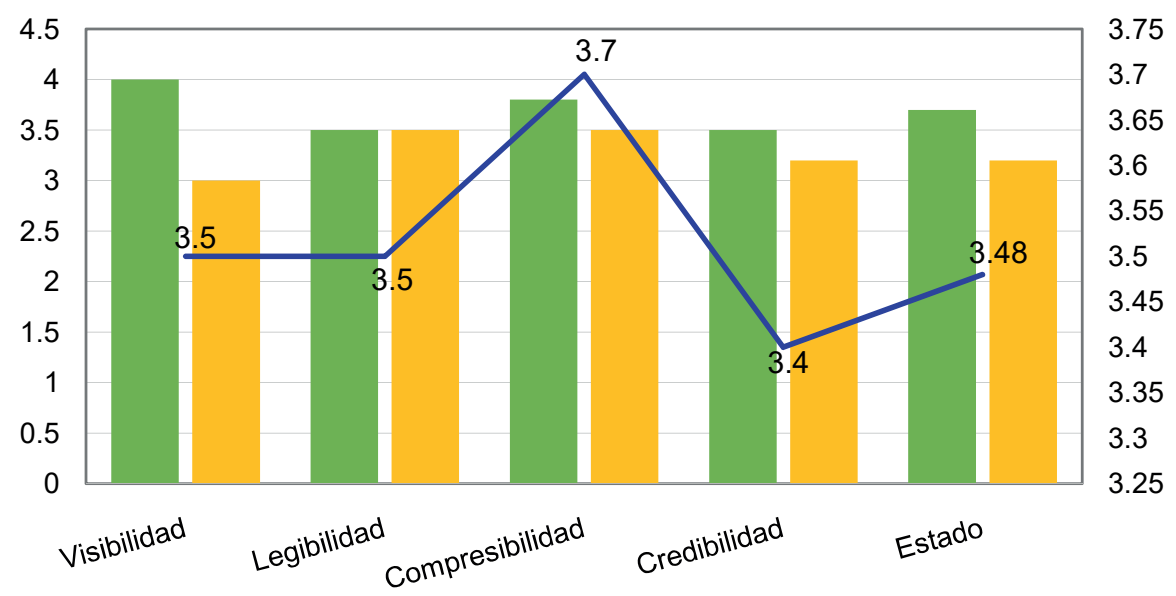

Señalización Vertical Señalización Horizontal $\longrightarrow$ Resultados Finales

Figura 6. La señalización en la troncal Avenida Caracas

\section{DISCUSIÓN}

La señalización tanto vertical como horizontal es un elemento fundamental que influye en la seguridad vial de los sistemas de transporte público de pasajeros tipo BRT, siempre y cuando estas cumplan con las características básicas de visibilidad, legibilidad, comprensibilidad y credibilidad, y se conserven en buen estado, con calificaciones mínimo de cuatro puntos, de acuerdo a la escala propuesta. En nuestro caso, se obtuvo una calificación promedio de 3,48 puntos, que en términos generales es aceptable. No obstante, esto indica que se debe mejorar el estado de la señalización para que esta llegue a ser buena o muy buena, especialmente la horizontal o las marcas en el pavimento para que sean más visibles y creíbles, de tal manera que sea respetada por todos los actores de la movilidad que interactúan con el sistema y se minimicen los posibles accidentes que se puedan presentar en el principal corredor troncal de los buses articulados de la ciudad capital.

En las evaluaciones y recorridos in situ, se pudo evidenciar que algunos operadores de los buses del sistema no respetan ciertas señales de tránsito corroborando algunas de las causas o hipótesis de los accidentes que se presentan en el sistema Transmilenio (Véase Figura 7). 


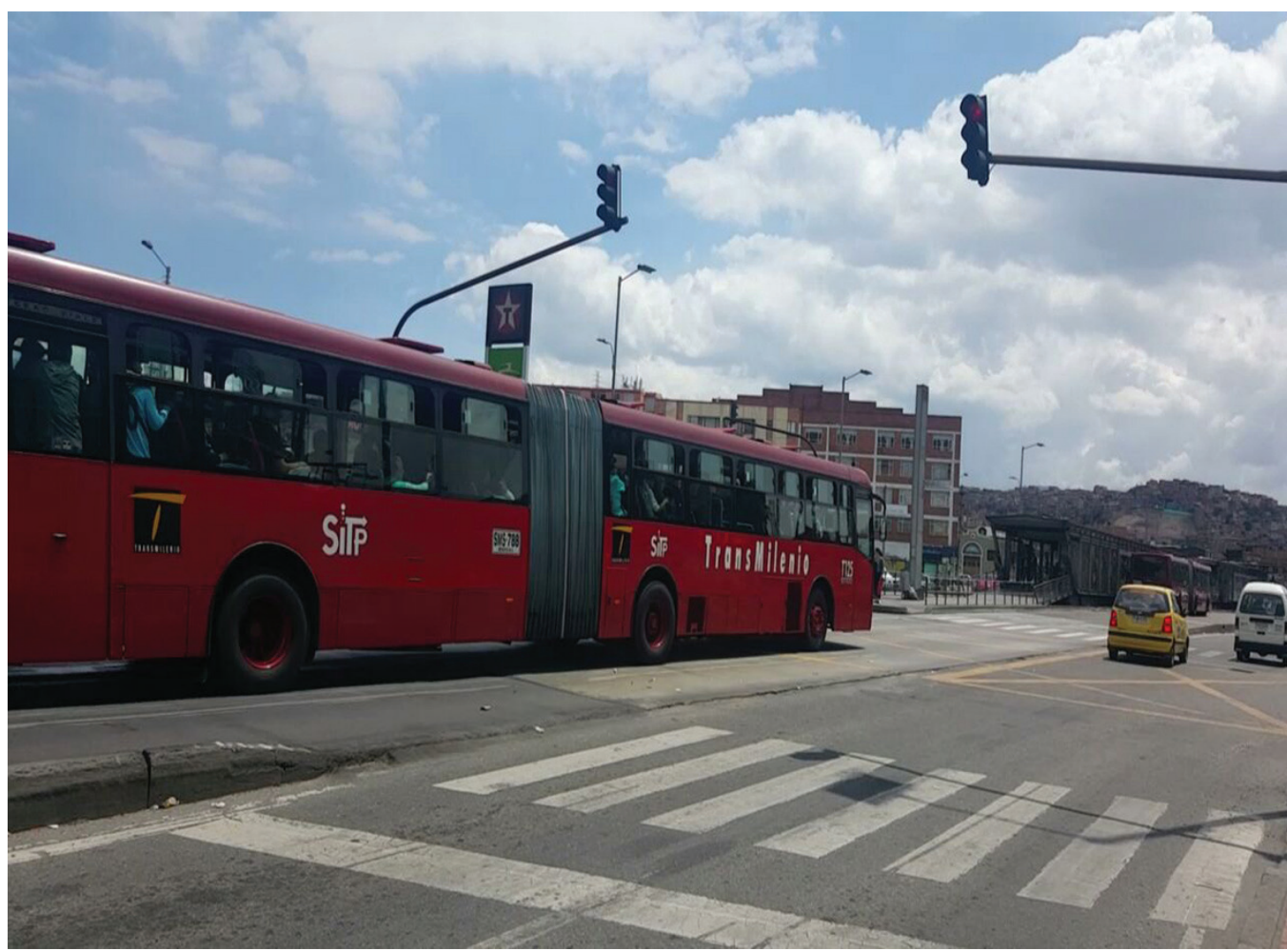

Figura 7. Desobediencia de señal semafórica en la troncal de la Ave. Caracas.

\section{CONCLUSIONES}

La señalización influye en los eventos o percances viales en la medida que estas no tengan un adecuado mantenimiento y reposición, para que cumplan con las funciones de guiar y orientar a los conductores de los buses del sistema Transmilenio y el resto de usuarios de la movilidad que circulan por el corredor vial. De ahí la necesidad de asignar los recursos suficientes para que en materia de señalización se responda a los estándares más altos y a su vez, se prolonge su conservación.

En Colombia, la construcción de corredores viales para la movilidad urbana y la señalización vial no ha sido la prioridad en las asignaciones presupuestales por parte de las entidades encargadas del mantenimiento y rehabilitación; pues ha sido evidente el incremento de la accidentalidad, y conlleva a la implementación de políticas públicas que posicionen la señalización, como uno de los elementos de la infraestructura que más incide en la seguridad vial. La señalización vertical y horizontal de la infraestructura del sistema Transmilenio deberá ser renovada y actualizada de acuerdo con los nuevos lineamientos de señalamientos introducidos en el recién expedido Manual de señalización vial: Dispositivos uniformes para la regulación del tránsito en calles, carreteras y ciclorutas de Colombia. Esto porque, las características de visibilidad, legibilidad, comprensibilidad y credibilidad no son las mejores, y en la mayoría de los casos las señales son desobedecidas constantemente por los diferentes actores de la movilidad que interactúan con el sistema de transporte; incluidos los operadores de las rutas troncales que transitan por la avenida Caracas.

Así pues, la seguridad es un elemento fundamental como indicador de la calidad o el nivel de servicio de los sistemas BRT. Por ello, el sistema Transmilenio tiene como propósito reducir los eventos viales que se han presentado en los últimos años, para lo cual se están implementando acciones como la reprogramación y reducción del número de rutas expresas como fáciles (paran en todas las estaciones) y hacer una intervención integral en la infraestructura del corredor troncal con mayor demanda de todo el sistema, donde la señalización, la semaforización, el pavimento y las intersecciones han sido elementos que se han considerado como prioritarios. 


\section{REFERENCIAS BIBLIOGRÁFICAS}

1. Asprilla, Y. \& Rey E. (2012). La implementación del Sistema Integrado de Transporte Público (SITP) de Bogotá y sus retos en el futuro. Tecnogestión 9 (1), 26-40. Recuperado 20 noviembre 2015 de: http://revistas.udistrital.edu.co/ojs/index.php/tecges /article/view/5649/7167

2. Chan, M. (2011). Comunicado de prensa: Unidos para frenar las muertes y los traumatismos causados por el tránsito, Organización Mundial de la Salud, EE.UU. Recuperado 3 enero 2016 de: http://www.who.int/mediacentre/news/releases/2011/road_safety_20110506/es/

3. Congreso de Colombia (1993). Ley 105 sobre la Planeación del Sector Transporte en Colombia. Diario Oficial No. 41.158. Recuperado 12 diciembre 2015 de: http:// www. secretariasenado.gov.co/senado/basedoc/ley_0105_1993.html

4. Duarte F. \& Rojas F. (2012). Intermodal Connectivity to BRT: a Comparative Analysis of Bogotá and Curitiba. Journal of Public Transportation, 15 (2), 1-18. Recuperado 4 marzo 2016 de: http://www.nctr.usf.edu/wp-content/uploads/2012/07/JPT15.2Duarte.pdf

5. El Universal (2013). Cuatro heridos por accidente de TransMilenio al norte de Bogotá. Recuperado 15 mayo 2015 de: http://www.eluniversal.com.co/colombia/ bogota/cuatro-heridos-por-accidente-de-transmilenio-al-norte-de-bogota-139475

6. Fondo de Prevención Vial (2014). Guía de lineamientos en seguridad vial y peatonal para diseño y operación de sistemas de transporte masivo en buses. Recuperado 12 mayo 2015 de: http://clementinagrafica.blogspot.mx/2014/05/guia-de-lineamientos-en-seguridad-vial.html

7. Gallardo, V. (2014). Lectura en señales de tránsito: Una revisión al impacto de la tipografía en las personas. Recuperado marzo 262016 de: https://www.academia. edu/ 21451828/Lectura_en_se\%C3\%B1ales_de_tr\%C3\%A1nsito_Una_revisi\%C3\%B3n_al_impacto_de_la_tipograf\%C3\%ADa_en_las_personas_espa\%C3\%B1ol_

8. Global BRT Data. (2016). Datos de Sistemas prioritarios de autobús de tránsito rápido, WRI-Brasil, EMBARQ-IEA, SIBRT. Recuperado 2 junio 2015 de: http:// brtdata.org

9. Google Earth (2016). Imágenes del corredor troncal del sistema Transmilenio, Ave Caracas, Colombia. Recuperado 12 febrero 2016 de: https://www.google.com. $\mathrm{mx} /$ intl/es/Earth

10. International Road Assessment Programme - IRAP (2009). Metodología calificación por estrellas para vías más seguras. Londres. Disponible en http://www.irap. org/en/about-irap-3/methodology/category/10-previous-model-methodology-papers

11. Jimeno, Berceruelo, M. L. (2013). Seguridad vial: Influencia de la señalización vertical. Revista técnica de la asociación española de Carreteras, (190), 41-49. Recuperado de 25 marzo 2016 de: http://www.afasemetra.com/doc/Articulos/CARRETERAS\%20190\%20AEC.pdf

12. Lindau, L., Hidalgo D. y Facchini, D. (2010). Curitiba, the cradle of Bus Rapid Transit. Built Environment, 36, (3), 274-282. Recuperado 17 noviembre 2015 de: http:// www. sibrtonline.org/downloads/built-environment-curitiba-oct19 4db0b5ac230da.pdf

13. Márquez, E. (2011). Señales: Características de Visibilidad y Legibilidad. Instituto de Diseño de Valencia. España. Recuperado 25 marzo 2016 de: http://wiki.ead. pucv. cl/images/5/51/Se\%C3\%B1ales_Caracter\%C3\%ADsticas_de_Visibilidad_y_Legibilidad.pdf

14. Ministerio de Transporte (2015). Manual de señalización vial: Dispositivos para la regulación del tránsito en calles, carreteras y ciclorutas de Colombia. Recuperado 11 mayo 2016 de: https://www.mintransporte.gov.co/Documentos/documentos_del_minis terio/Manuales/manuales_de_senalizacion_vial

15. Ministerio de Transporte (2014)- Plan Nacional de seguridad Vial 2013-2021. Recuperado diciembre de 2015 https://culturavial.files.wordpress.com/2014/01/ consulta_plan_nacional_de_seguridad_vial_colombia_2013-2021.pdf

16. Organización de Estados Americanos (1991). Manual Interamericano de dispositivos para el Control del tránsito en calles y carreteras. Recuperado 2 abril 2016 de: http://200.35.84.131/portal/bases/marc/texto/013431-Libro.pdf

17. Organización de Naciones Unidas (1949). Primera asamblea de países miembros, con el fin de discutir una propuesta para la unificación de las señales de tránsito. Ginebra, Suiza.

18. Consejo Económico y Social (1955). Aprobación del sistema mundial de señales. 1. New York. EE.UU

19. Organización Mundial de la Salud-OMS (2015). Informe mundial sobre la situación de la seguridad vial. Recuperado 27 noviembre 2015 de: http://www.who.int/ violence_injury_prevention/road_safety_status/2015/en/

20. Rune, E. Hoye, A., Vaa, T. \& Sorensen, M. (2013). Manual de Medidas de Seguridad Vial. España: Fundación Mapfre.

21. Secretaria de Comunicaciones y Transporte (2014). Manual de señalización vial y dispositivos de seguridad. Dirección General de servicios técnicos, sexta edición. México. Recuperado 11 mayo 2016 de: http://sct.gob.mx/fileadmin/DireccionesGrales/DGST/Manuales/NUEVOSENAL AMIENTO/ manualSenalamientoVialDispositivosSeguridad.pdf

22. Secretaria Distrital de Movilidad (2016). Respuesta oficio solicitud sobre Informe Accidentalidad sistema Transmilenio, Colombia. Recuperado 2 abril 2016 de: www. movilidadbogota.gov.co/

23. Tignor, S (1999). Traffic Control devices. Washington, D.C.: Institute of Transportation Engineers.

24. Transmilenio (2014) Informe de Gestión Transmilenio. Recuperado 4 abril 2016 de: http://www.transmilenio.gov.co/sites/default/files/informe_de_gestion_2014_ version_final_1.4_22_de_abril_de_2015.pdf 